\title{
Assessing the accuracy of self-reporting HIV testing behaviour in Houston/Harris County, Texas
}

\author{
Hafeez Rehman ${ }^{1}$, Salma Khuwaja ${ }^{1}$, Zuhair Siddiqui ${ }^{1}$, Karen Chronister $^{2}$ \\ ${ }^{1}$ Houston Health Department, Houston, United States \\ ${ }^{2}$ The Kirby Institute, UNSW, Australia
}

\begin{abstract}
Introduction: In 2006, the Centers for Disease Control and Prevention (CDC) began directly estimating human immunodeficiency virus (HIV) incidence based upon the Serologic Testing Algorithm for Recent HIV Seroconversion (STARHS) and individuals' testing and treatment history, collected as part of the HIV Incidence Surveillance (HIS) system. The algorithm relies largely upon individuals' self-reported testing history. The primary objective of this study is to describe the methodology and procedures used to assess the similarities between self-reported and medical record data on HIV status and the dates of first positive and last negative tests.

Material and methods: The testing history from the individuals is used in combination with the latest laboratory assay tests to obtain a direct population-based estimate of HIV incidence. Understanding how accurate the self-report testing information is will help in estimating HIV incidence. Partnerships were made with the medical clinics and Counselling and Testing facilities, and the participants were recruited from the patients attending these facilities. Participants were interviewed to ascertain the self-report of HIV test, HIV status, the date and location of the most recent HIV-negative test and the first HIV-positive test. Medical record abstraction was done after participants' authorisation to compare the accuracy of self-report testing data.

Results: The participants' response rate was $83.7 \%$, and the most common reason of not participating was lack of interest in the study. Data analysis is reported by CDC and Houston Health Department in a separate article.

Conclusions: The methodology and procedures adopted in this study can be adopted, replicated, and improved in future studies exploring self-report accuracy.
\end{abstract}

HIV AIDS Rev 2019; 18, 1: 50-56 DOI: https://doi.org/10.5114/hivar.2019.84201

Key words: HIV testing, self-report, HIV/AIDS.

\section{Introduction}

Human immunodeficiency virus (HIV) surveillance has been an evolving process since its initial outbreak in the 1980s.
Before advances in treatment with highly active antiretroviral therapies (HAART) and a change in the case definition for acquired immunodeficiency syndrome (AIDS) in 1993, HIV incubation periods were shorter; the algorithm used to
Address for correspondence: Hafeez Rehman, Epidemiology, Houston Health Department, 8000 N Stadium Dr, 77054, Houston, United States, e-mail: hapheez@hotmail.com

\section{Article history:}

Received: 21.06.2018

Received in revised form: 31.08 .2018

Accepted: 06.02.2019

Available online: 28.03.2019
International Journal of HIV-Related Problem

HIV \& AIDS

R e vi e w 
estimate HIV incidence was derived from back-calculation models of AIDS rates [1-5]. As treatment improved throughout the 1990s, HIV incubation periods became longer, and its incidence became more difficult to estimate [6]. Estimates of annual HIV incidence have also been derived from cohort studies [7], but they were not useful for historical trends or population-based incidence [6].

Recent advancements in laboratory assays for HIV have enabled a new method of directly measuring HIV incidence. BED HIV-1 capture enzyme immunoassay (BED-CEIA) can differentiate newer infections from older infections [8]. It achieves this by measuring the ratio of anti-HIV IgG to total IgG; the ratio increases shortly after an HIV infection, indicating the infection is a recent one. From this, the serologic testing algorithm for recent HIV seroconversion (STARHS) was developed. Serologic Testing Algorithm for Recent HIV Seroconversion (STARHS) weighs individuals based on the probability of having been tested within one year of infection, the probability of an HIV-positive person receiving a BED-CEIA test, and the probability that the test classified the infection as "recent" [6]. Two different equations are used to estimate the probability of having been tested within one year of infection. One equation is for repeat testers who had their first positive, and one is for first-time testers who had their first positive. This behavioural information relies upon the self-report of testing and treatment history (TTH), which is collected from recently diagnosed cases as part of the HIV Incidence Surveillance (HIS) system [9]. Among repeat testers, the infection date was assumed to be uniformly distributed from the date of the most recent negative result to the date of the first positive result $[6,10]$. Based on these assumptions, determining the validity of self-reported information on HIV testing history is crucial to accurately estimating the incidence $[6-8,10]$. Moreover, the estimation model is sensitive to changes in HIV testing patterns, such as demonstrated by the implementation of the Centers for Disease Control and Prevention's (CDC) 2006 HIV testing recommendations $[10,11]$.

Many studies previously examined the accuracy of self-reported testing and disease screening [12-15]; few have examined self-reporting of HIV testing [16-20], and even fewer have examined self-reported testing with medical record reviews [21]. A common finding among studies examining self-reporting was "telescoping," where subjects recall testing to have occurred longer ago than it had actually occurred. The varying concordance, sensitivity, specificity, and kappa statistics in these studies suggests that recalling screening services for major diseases may be linked to a number of other factors. Furthermore, these varied results suggest that the validity of self-reported dates must be evaluated when they are relied upon for a number of purposes, including research, surveillance, and prevention.

Because the algorithm for directly estimating HIV incidence relies on self-reported testing history, and previous studies have shown discrepant findings of self-reported information, this study examined the reliability of self-reported HIV data in a diverse patient population in Houston,
Texas. We sought to simulate the TTH questions for the purposes of this study, in order to answer two fundamental research questions:

1. How accurately do individuals remember receiving a HIV test, and is this memory associated with sex, race/ ethnicity, age, or other variables; and,

2. Among those individuals who have received a HIV test, how accurately do they remember the last HIV testing date, and is this associated with the length of time elapsed, sex, race/ethnicity, age, or other variables? We also created several specific aims for the project:

1. Partner with at least one medical clinic and at least one publicly funded confidential counselling, testing, and referral (CTR) facility to recruit participants for the study.

a. Partnered with Legacy Community Health Services (LCHS), Harris Health System (HHS), and the City of Houston Health Department (HHD) as data collection sites.

b. HHS is an HMO; HHD and LCHS are publicly funded confidential CTR services. Historically, these facilities have diagnosed a significant portion of Houston's new HIV diagnoses and partnered with HHD for research purposes.

2. Develop interview questions related to previous HIV testing, results, and dates.

Interview questions were developed by HHD with consultation from the CDC. The instrument is a 12 -item self-administered questionnaire, titled Assessing the Accuracy of Self-Report HIV Testing Behaviour Questionnaire. Items 1-4 covered demographic information, such as age, sex, ethnicity, and race. Item 12 asks about other medical providers the participant visited in the last three years. Items 5-11 address previous HIV testing and results. The questionnaire used a skip pattern to differentiate between participants to claim to have never been tested and those who have been previously tested. The questionnaire then further differentiates between participants who have or have not had a positive result. If there is a positive result, the participant is asked to provide the month, year, and location of the first time he or she ever tested positive. The questionnaire also differentiates between those who have or have not had a negative test result. If there is a negative test result, the participant is asked to provide the month, year, and location of the first time he or she ever tested negative. The study team originally planned to administer the questionnaire electronically, but it soon became evident that recruitment and quality assurance could be done more easily in paper format.

3. Conduct a medical record abstraction to determine if a patient had an HIV test, current HIV status, and the date and location of the most recent negative HIV test for individuals who have had at least one HIV test, and the date and location of the first positive test among participants who are HIV positive.

Medical records were abstracted following the recruitment, consent, and interview of study participants. Abstractions 
were completed using five electronic sources, the interview location, and any other providers that the participant indicated on their form. Both electronic and physical records were accessed for HHD and LCHS to ensure accuracy.

4. Interview participants to ascertain self-reports of whether the participants have had an HIV test, HIV status, the date and location of the most recent negative HIV test for those who have had at least one HIV test, and the date and location of the first positive HIV test for HIV-positive individuals.

Surveillance investigators solicited participation in the waiting rooms and lobbies of participating clinics. The study was briefly explained, and if the client was interested in participating, the client was given the option of completing the interview in a private setting. The study was then explained in detail, including the Informed Consent to Join a Research Study and the Authorisation for Release of Protected Health Information. After these forms were completed, the participant was given a selfadministered 12-item study questionnaire in either electronic or paper format. After completing the questionnaire, the team member conducted quality assurance for accuracy and completeness. Finally, the participant was given a $\$ 10$ gift card to a grocery store as an incentive to participate, and the participant and interviewer completed the Incentive Acknowledgement Form.

\section{Material and methods}

This study contained several steps: interviewing, medical record abstraction, data entry, and analysis. The interview involved recruitment, obtaining informed consent and release of medical information, administering the questionnaire, and distributing the incentive to the participant. All documents related to the interview process were developed by the investigators and received IRB approval from the Committee for the Protection of Human Subjects of the University of Texas Health Science Center at Houston, the Harris Health System Research and Sponsored Programs, and the HHD Investigative Review Committee. Participants were recruited from the waiting rooms and lobbies of participating clinics by displaying and distributing a flyer with a brief overview of the study. A brief overview of the study was given, and if the client showed interest, he or she was taken to a private area for the consent process and questionnaire. The study was described in detail, and the participant was given an Informed Consent to Join a Research Study and the Authorization for Release of Protected Health Information form. Once these forms were completed, the participant was given the opportunity to have any questions answered and then given a self-administered 12-item Assessing the Accuracy of Self-Report of HIV Testing Behaviour Questionnaire in either electronic or paper format. Once the questionnaire was completed, a team member conducted quality assurance by reviewing the document for accuracy and completeness. The participant was then given a $\$ 10$ gift card to a grocery store as a participation incentive, and the interviewer completed the Incentive Acknowledgement Form. Completed forms were placed in a passcode-protected briefcase and transported from the data collection site to a secure, locked file in a room, accessible only by HHD Bureau of Epidemiology staff members, prior to data entry. Once data entry was completed, the forms were placed back into the locked file and limited-access room.

All clients who were aged 18 years and over when interviewed, who accessed medical care from April 2012 through May 2013 at the participating clinics, and who gave written, informed consent alongside a written authorisation for the release of medical information were eligible for the study. Clients were recruited using a convenience, or accidental, sampling method stratified by facility. Recruitment was targeted at the three systems that had a diverse population and the highest number of HIV diagnoses in 2007 (unpublished data): LCHS, HHS, and HHD clinics. LCHS is a non-profit, Federally Qualified Health Centre with nine locations across the greater-Houston Area that function as confidential counselling, testing, and referral (CTR) facilities; we recruited participants from four of these locations. The Harris Health System is a public health system that serves Harris County through three hospitals and 22 health centres, providing a health maintenance organisation (HMO) for its patients; we utilised 13 of their primary care facilities in Houston. Finally, the HHD has four publicly funded confidential CTR facilities, all of which were used for this study.

Medical records were obtained after participants consented to and completed the study. Investigators used the Medical Record Abstraction form to compile medical records from five electronic sources, the interview location's medical records, and any other providers, clinics, or hospitals that the participant indicated on the Authorisation for Release of Protected Health Information. The five electronic sources were Harris Health System's Epic electronic medical record system, LCHS's Epic electronic medical record system, HHD's Cyber Lab laboratory record system, the Electronic Client-Level Integrated Prevention System (ECLIPS) walk-up testing database, and Harris Health's walk-up testing database.

The Harris Health System has used only electronic medical records since 2002, so their Epic system was used to access participant's medical records. HHD and LCHS use physical and electronic medical records, so for participants enrolled in these systems, both electronic and paper charts were reviewed for accuracy and completeness. ECLIPS's and Harris Health's walk-up testing databases were also abstracted from. If participants authorised the release of medical records from another clinic not enrolled in the study, data collection staff contacted the physician, clinic, hospital, or institution in order to conduct a medical record review.

The date of the first positive HIV test and the date of the last negative HIV test was retrieved from authorised locations. These dates were abstracted from the medical records by trained data-collection personnel; the first positive test date 
was defined as the earliest date of a positive laboratory test (e.g. an enzyme immunoassay [EIA], a Western blot assay, or a rapid antibody test), and the last negative test date was defined as the most recent date of a negative laboratory test. The medical record was the "gold standard", but for the purposes of this study, medical record notes were not considered valid sources for these dates. The data collection staff completed one Medical Record Abstraction Form for each electronic and physical record accessed. Re-abstraction was completed on $5 \%$ of the records for quality assurance, and a $90 \%$ agreement was deemed acceptable for research purposes.

The participant's name was linked to a unique participant code on a single, separate, encrypted, and secure database for de-identification. The Information from the Authorisation for Release of Health Information, Assessing the Accuracy of Self-Report of HIV Testing Behaviour Questionnaire, Incentive Acknowledgement Form, and Medical Record Abstraction Form were filed using the unique participant code. The providers indicated on the Authorisation for Release of Health Form were entered into a Microsoft Access database to track the specific locations authorised for medical record abstraction by each participant and to track the progress of these medical record abstractions. The information collected was entered into a database in Questionnaire Development System, Version 2.6.1 (QDS) (NOVA Research Company: Bethesda, MD) for storage. Information from the Incentive Acknowledgement Form was entered into a Microsoft Excel spreadsheet to track the distribution of incentives. Medical record information abstracted using the Medical Record Abstraction Form was entered into a separate Microsoft Access database. The databases for the questionnaires and medical record abstractions were later joined into a single Microsoft Access database for cleaning and later analysis. Quality assurance of data entry was conducted by re-entry of $10 \%$ of the questionnaires and medical record abstractions, with a $90 \%$ agreement deemed acceptable for research purposes.

The data from the questionnaire and the medical record abstractions were imported into SAS, Version 9.3 (SAS) (SAS Institute: Cary, NC). SAS was used to associate the two datasets, develop necessary variables, and run the descriptive and statistical analysis.

\section{Results}

A total of 1897 potential participants were approached throughout the study, and 301 (15.8\%) of them refused to participate. From April 2012 to May 2013, the study team conducted 1597 participant interviews across the three partner health systems. Throughout the data collection period, there were 23 duplicate participant interviews and 19 interviews were accidentally destroyed, leading to a final participant count of 1555. Approximately one-fourth of the participants $(n=378)$ authorised a total of 456 other providers or facilities from which to abstract medical records. In total, we obtained about two-thirds of these records $(n=292)$. Among those we were unable to obtain were 27 records from the Texas Department of Criminal Justice. Unfortunately, we were not able to locate any records for 52 participants, which equates to a $96.7 \%$ abstraction rate.

In total, there were 1503 participants included in the data analysis. The data analysis was completed by a team of scientists from CDC and HHD and reported in a separate study [22].

All the participants were recruited from the three health systems ( 21 clinics total) (Table 1 ). The study population had a higher percentage $(58.3 \%)$ of females than males; this was due to the disproportionate population accessing care from HHD (74.6\% female) and the use of convenience sampling in these clinics. For similar reasons, our study population had a higher percentage of black, non-Hispanic (47.0\%), and Hispanic (35.8\%) participants. For the purposes of this study, races and ethnicities other than black, white, and Hispanics were grouped together with participants who identified multiple races or ethnicities; this other/multiple race/ethnicity category accounted for only $3.6 \%$ of our participants.

Throughout the study, we had 301 non-responders, giving an $83.7 \%$ response rate (Table 2 ).

The sex, race/ethnicity, and age of the non-responders varied significantly from the study population ( $p$-values of $0.0120,<0.0001$, and 0.0264 , respectively). The nonresponders had a higher proportion of male, Hispanic, white, 30-39-year-old, and 60+ year old individuals, compared with the study population. Common reasons for non-response included: lack of interest in participating in a study (61.0\%); lack of time/appointment at the health centre (17.9\%); and discomfort with disclosing/releasing information (12.8\%).

Limitations: A limitation of the study was the inability to obtain all of medical records. We were unable to obtain the records from the Texas Department of Criminal Justice because they required further IRB approval. The other records we were unable to review were due to ambiguity or unclear indication on the authorisation form, refusal of the provider or facility to allow our team to review, the provider did not have a record for the patient, or the provider or facility had closed. Another limitation encountered was the inability to match testing records from ECLIPS and Harris Health databases with our study participants, if the participant had elected for confidential testing; the study team determined this to be an impassable but very minor limitation.

\section{Discussion}

The overarching purpose of the research project was to compare what people remember about HIV tests with what is recorded in their medical records.

Testing history from HIV positive individuals is used in conjunction with results from STARHS to provide a direct population-based estimate of HIV incidence [6]. Understanding how accurate this information is will guide the use of this information for estimating HIV incidence. Furthermore, HIV incidence measurements are utilised to monitor the epidemic and to inform and guide policy, programs, 
Table 1. Demographics of participants in the Assessing the Accuracy of Self-Report of HIV Testing history

\begin{tabular}{|c|c|c|c|c|c|c|c|c|}
\hline \multirow[t]{2}{*}{ Characteristic } & \multicolumn{2}{|c|}{$\begin{array}{l}\text { HHS (HMO) } \\
(n=628)\end{array}$} & \multicolumn{2}{|c|}{$\begin{array}{c}\text { HHD (CTR) } \\
(n=456)\end{array}$} & \multicolumn{2}{|c|}{$\begin{array}{l}\text { LCHS (CTR) } \\
(n=471)\end{array}$} & \multicolumn{2}{|c|}{$\begin{array}{c}\text { Total } \\
(n=1555)\end{array}$} \\
\hline & No. & $\%$ & No. & $\%$ & No. & $\%$ & No. & $\%$ \\
\hline \multicolumn{9}{|l|}{ Gender } \\
\hline Male & 304 & 48.4 & 116 & 25.4 & 229 & 48.6 & 649 & 41.7 \\
\hline Female & 324 & 51.6 & 340 & 74.6 & 242 & 51.4 & 906 & 58.3 \\
\hline \multicolumn{9}{|l|}{ Race/Ethnicity } \\
\hline Black, non-Hispanic & 292 & 46.5 & 262 & 57.5 & 177 & 37.6 & 731 & 47.0 \\
\hline Hispanic & 214 & 34.1 & 161 & 35.3 & 182 & 38.6 & 557 & 35.8 \\
\hline Other/Multiple & 22 & 3.5 & 18 & 3.9 & 16 & 3.4 & 56 & 3.6 \\
\hline White, non-Hispanic & 100 & 15.9 & 15 & 3.3 & 96 & 20.4 & 211 & 13.6 \\
\hline \multicolumn{9}{|l|}{ Age at study consent* } \\
\hline $18-24$ & 20 & 3.2 & 108 & 23.7 & 28 & 5.9 & 156 & 10.0 \\
\hline $25-29$ & 29 & 4.6 & 107 & 23.5 & 60 & 12.7 & 196 & 12.6 \\
\hline $30-34$ & 43 & 6.8 & 73 & 16.0 & 52 & 11.0 & 168 & 10.8 \\
\hline $35-39$ & 65 & 10.4 & 51 & 11.2 & 59 & 12.5 & 175 & 11.3 \\
\hline $40-44$ & 60 & 9.6 & 48 & 10.5 & 65 & 13.8 & 173 & 11.1 \\
\hline $45-49$ & 107 & 17.0 & 32 & 7.0 & 67 & 14.2 & 206 & 13.2 \\
\hline $50-54$ & 129 & 20.5 & 13 & 2.9 & 69 & 14.6 & 211 & 13.6 \\
\hline $55-59$ & 78 & 12.4 & 10 & 2.2 & 38 & 8.1 & 126 & 8.1 \\
\hline $60+$ & 97 & 15.4 & 14 & 3.1 & 33 & 7.0 & 144 & 9.3 \\
\hline
\end{tabular}

Age at first positive*

\begin{tabular}{l|c|c|c|c|c|c|c|c}
\hline $18-24$ & 46 & 16.1 & 0 & 0.0 & 29 & 20.0 & 75 & 17.3 \\
\hline $25-34$ & 74 & 25.9 & 3 & 100.0 & 59 & 40.7 & 136 & 31.3 \\
\hline $35-44$ & 91 & 31.8 & 0 & 0.0 & 40 & 27.6 & 131 & 30.2 \\
\hline $45-54$ & 57 & 19.9 & 0 & 0.0 & 12 & 8.3 & 69 & 15.9 \\
\hline $55+$ & 18 & 6.3 & 0 & 0.0 & 5 & 3.4 & 23 & 5.3 \\
\hline
\end{tabular}

Age at last negative*

\begin{tabular}{l|c|c|c|c|c|c|c|c}
\hline $18-24$ & 8 & 7.2 & 73 & 31.5 & 20 & 15.9 & 101 & 21.5 \\
\hline $25-34$ & 26 & 23.4 & 101 & 43.5 & 50 & 39.7 & 177 & 37.7 \\
\hline $35-44$ & 32 & 28.8 & 40 & 17.2 & 27 & 21.4 & 99 & 21.1 \\
\hline $45-54$ & 29 & 26.1 & 14 & 6.0 & 22 & 17.5 & 65 & 13.9 \\
\hline $55+$ & 16 & 14.1 & 4 & 1.7 & 7 & 5.6 & 27 & 5.8 \\
\hline
\end{tabular}

HHS - Harris Health System, HHD - Houston Health Department, LCHS - Legacy Community Health Services, HMO - Health Maintenance Organization, CTR - Counselling, Testing, and Referral

${ }^{*}$ Calculated from dates provided during interview.

and practices in the United States. Therefore, it is crucial to provide the most accurate and reliable tools for estimating incidence.

Our findings will be useful in establishing modifications to the HIV incidence estimate calculation. Because we have shown that individuals are largely unable to recall whether they have previously been tested, the algorithm currently used may need to be modified to account for this discrepancy in self-reporting. Currently, recently diagnosed individuals are categorised as first-time testers or repeat testers, then these two groups have different calculations in the determination of incidence [6]. Furthermore, showing that recall of the month and year of participants' last negative can be inaccurate and that differences exist among various demographics, the reliance on this measure in the calculations used for repeat testers will need to be re-evaluated and adjusted. Finally, this study will guide future research into the accuracy and reliability of self-reported testing and treatment history, inform about alternative methods of estimating the incidence of HIV infection in the United States, 
Table 2. Demographics of non-responders in the Assessing the Accuracy of Self-Report of HIV Testing history

\begin{tabular}{|c|c|c|c|c|}
\hline \multirow[t]{2}{*}{ Characteristic } & \multicolumn{2}{|c|}{$\begin{array}{l}\text { Non-response } \\
\quad(n=301)\end{array}$} & \multicolumn{2}{|c|}{$\begin{array}{l}\text { Study population } \\
\quad(n=1555)\end{array}$} \\
\hline & No. & $\%$ & No. & $\%$ \\
\hline \multicolumn{5}{|l|}{ Gender } \\
\hline Male & 147 & 48.8 & 649 & 41.7 \\
\hline Female & 154 & 51.2 & 906 & 58.3 \\
\hline \multicolumn{5}{|l|}{ Race/Ethnicity } \\
\hline Black, non-Hispanic & 100 & 33.2 & 731 & 47.0 \\
\hline Hispanic & 125 & 41.5 & 557 & 35.8 \\
\hline Other/Multiple & 5 & 1.7 & 56 & 3.6 \\
\hline White, non-Hispanic & 71 & 23.6 & 211 & 13.6 \\
\hline \multicolumn{5}{|l|}{ Age at study consent* } \\
\hline $18-29$ & 64 & 21.3 & 352 & 22.6 \\
\hline $30-39$ & 85 & 28.2 & 343 & 22.1 \\
\hline $40-49$ & 66 & 21.9 & 379 & 24.4 \\
\hline $50-59$ & 51 & 16.9 & 337 & 21.7 \\
\hline $60+$ & 35 & 11.6 & 144 & 9.3 \\
\hline
\end{tabular}

${ }^{*}$ Calculated from dates provided during interview.

and advance recommendations for public health policy and practice in HIV surveillance.

From the results of the research project, CDC will evaluate the validity of using self-reported HIV testing history information for directly estimating HIV incidence. Currently, testing and treatment history information from HIV-positive individuals is used in conjunction with results from STARHS to provide a direct population-based estimate of HIV incidence [6]. This estimation is used for research, trend analysis, public policy, prevention, interventions, and many other purposes. Furthermore, we were able to identify systematic recall bias in the overall population and by sub-population, which will assist with the calculations and assumptions made in the general population and within particular demographics in this algorithm. Through showing that there seems to be consistent inaccuracies in self-reported information, we hope to improve the ability to directly estimate this crucial measure. By improving this measure, we will subsequently be improving public health policy, surveillance practices, research endeavours, and prevention practices.

Because this is the first study on this specific topic, we hope that the methodology and procedures used can be employed, replicated, and/or improved upon for future studies in this area. Our study was limited in scope and size, so it will be important to replicate and expound upon our findings. Furthermore, this study will contribute to the growing literature on the accuracy and reliance of self-reported medical information in general. This literature is used to inform healthcare practices, research methodologies, surveillance practices, and many other areas.

\section{Acknowledgements}

The authors would like to thank the following clinics and individuals for their participation in the study and contribution with data collection:

Participating facilities from Harris Health System: Acres Home Health Center, Aldine Health Center, Baytown Health Center, Casa de Amigos Health Center, E.A. Squatty Lyons Health Center, El Franco Lee Health Center, Gulfgate Health Center, Martin Luther King Jr Health Center, Northwest Health Center, Settegast Health Center, Strawberry Health Center, Thomas Street Health Center, Vallbona Health Center.

Participating facilities from Houston Health Department: La Nueva Casa de Amigos Health Center, Northside Health Center, Sharpstown Health Services, Sunnyside Health Center.

Participating facilities from Legacy Community Health Services: Baker-Ripley Clinic, Lyons Clinic, Montrose Clinic, Southwest Clinic.

Staff from Houston Health Department: Veronica Anderson, Tristan Broussard, Shirley Chan, Jeff Meyer, Eileen Collet, Jasmin Prudon, Kellie Watkins.

Staff from the Centers for Disease Control and Prevention: Prejean Joseph, Jane Kelly.

This work was supported by Centers for Disease Control and Prevention through cooperative agreement under Grant number 5U01PS001552 to Houston Health Department.

\section{Conflict of interest}

The authors declare no potential conflicts of interest with respect to the research, authorship, and/or publication of this article. 


\section{References}

1. Biggar RJ, Rosenberg PS. HIV infection/AIDS in the United States during the 1990s. Clin Infect Dis 1993; 17 Suppl 1: S219-223.

2. Brookmeyer R. Reconstruction and future trends of the AIDS epidemic in the United States. Science 1991; 253: 37-42.

3. Karon JM, Khare M, Rosenberg PS. The current status of methods for estimating the prevalence of human immunodeficiency virus in the United States of America. Stat Med 1998; 17: 127-142.

4. Rosenberg PS. Backcalculation models of age-specific HIV incidence rates. Stat Med 1994; 13: 1975-1990.

5. Rosenberg PS. Scope of the AIDS epidemic in the United States. Science 1995; 270: 1372-1375.

6. Hall HI, Song R, Rhodes P, et al. Estimation of HIV incidence in the United States. JAMA 2008; 300: 520-529.

7. Karon JM, Fleming PL, Steketee RW, De Cock KM. HIV in the United States at the turn of the century: an epidemic in transition. Am J Public Health 2001; 91: 1060-1068.

8. Parekh BS, Kennedy MS, Dobbs T, et al. Quantitative detection of increasing HIV type 1 antibodies after seroconversion: a simple assay for detecting recent HIV infection and estimating incidence. AIDS Res Hum Retroviruses 2002; 18: 295-307.

9. CDC. Revised surveillance case definitions for HIV infection among adults, adolescents and children aged $<18$ months and for HIV infection and AIDS among children aged 18 to $<13$ years - United States, 2008. MMWR Morb Mortal Wkly Rep 2008; 57(RR-10): 1-12.

10. Prejean J, Song R, Hernandez A, et al. Estimated HIV incidence in the United States, 2006-2009. PLoS One 2011; 6: e17502.

11. CDC. Revised recommendations for HIV testing of adults, adolescents, and pregnant women in health-care settings. MMWR Morb Mortal Wkly Rep 2006; 55(RR-14): 1-17.

12. Chan EC, Vernon SW, Ahn C, Greisinger A. Do men know that they have had a prostate-specific antigen test? Accuracy of selfreports of testing at 2 sites. Am J Public Health 2004; 94: 1336-1338.

13. Hall HI, Van Den Eeden SK, Tolsma DD, et al. Testing for prostate and colorectal cancer: comparison of self-report and medical record audit. Prev Med 2004; 39: 27-35.

14. Volk RJ, Cass AR. The accuracy of primary care patients' self-reports of prostate-specific antigen testing. Am J Prev Med 2002; 22: 56-58.

15. Zapka JG, Bigelow C, Hurley T, et al. Mammography use among sociodemographically diverse women: the accuracy of self-report. Am J Public Health 1996; 86: 1016-1021.

16. Bignami-Van Assche S, Chao LW, Anglewicz P, et al. The validity of self-reported likelihood of HIV infection among the general population in rural Malawi. Sex Transm Infect 2007; 83: 35-40.

17. Fisher DG, Reynolds GL, Jaffe A, Johnson ME. Reliability, sensitivity and specificity of self-report of HIV test results. AIDS Care 2007; 19: 692-696.

18. McCusker J, Stoddard AM, McCarthy E. The validity of self-reported HIV antibody test results. Am J Public Health 1992; 82: 567-569.

19. Pedrana AE, Hellard ME, Guy R, et al. The difference in self-reported and biological measured HIV prevalence: implications for HIV prevention. AIDS Behav 2012; 16: 1454-1463.

20. Yudin MH, Barbara AM, Guenter D, et al. Medical records and women's self-report are not reliable sources for determining whether prenatal HIV testing was done. J Obstet Gynaecol Can 2006; 28: 867-872.

21. McCoy SI, Jones B, Leone PA, et al. Variability of the date of HIV diagnosis: a comparison of self-report, medical record, and HIV/ AIDS surveillance data. Ann Epidemiol 2010; 20: 734-742.

22. An Q, Chronister K, Song R, et al. Comparison of self-reported HIV testing data with medical records data in Houston, TX 2012-2013. Ann Epidemiol 2016; doi: 10.1016/j.annepidem.2016.02.013 [Epub ahead of print]. 